\title{
Longer is not better: nonconscious overstimulation reverses priming influences under interocular suppression
}

\author{
Antoine Barbot $\cdot$ Sid Kouider
}

Published online: 28 October 2011

(C) Psychonomic Society, Inc. 2011

\begin{abstract}
Previous research has shown that stimuli rendered invisible through masking can be sufficiently processed to induce nonconscious influences and facilitate subsequent recognition. However, masking paradigms are methodologically restricted such that stimuli cannot be presented for longer than a few tens of milliseconds, potentially restricting the strength of nonconscious influences. By adapting a masked face repetition priming paradigm to a recent interocular suppression method, we investigated whether longer periods of invisible prime stimulation lead to larger nonconscious influences on subsequent recognition. Surprisingly, we found that while brief periods of invisible prime stimulation result in classical facilitation priming, long periods of invisible stimulation lead to negative priming influences, reflecting impairment of subsequent recognition. In contrast, when the prime was visible, longer exposure resulted in classical facilitation effects, revealing qualitative differences between conscious and nonconscious processes. Altogether, the present findings reveal the existence of a nonconscious overstimulation cost, as well as an important dissociation between conscious and nonconscious processing.
\end{abstract}

Electronic supplementary material The online version of this article (doi:10.3758/s13414-011-0226-3) contains supplementary material, which is available to authorized users.

A. Barbot $\cdot$ S. Kouider

Laboratoire des Sciences Cognitives et Psycholinguistique, CNRS/EHESS/DEC-ENS,

Paris, France

\author{
A. Barbot $(\square)$ \\ Department of Psychology, New York University, \\ 6 Washington Place, 9th Floor, \\ New York, NY 10003, USA \\ e-mail: antoine.barbot@nyu.edu
}

Keywords Repetition priming · Interocular suppression . Nonconscious processing $\cdot$ Negative priming

\section{Introduction}

To what extent can perceptual information be processed in the absence of awareness? Nowadays, while the existence of nonconscious processing is definitely accepted, the debate has shifted to its extent and limits (Kouider \& Dehaene, 2007; Lin \& He, 2009). Masked priming experiments, in which a prime stimulus is rendered invisible by the joint use of brief presentations $(\sim 50 \mathrm{~ms})$ and backward masking, have been particularly helpful in demonstrating subliminal influences at behavioral and neural levels (Kouider \& Dehaene, 2007; Marcel, 1983). In this paradigm, the prime stimulus activates multiple representation levels, but its processing is interrupted by the mask prior to visual awareness. Importantly, this activation by the invisible prime provides a "head start" to the processing of subsequent visible targets, resulting in facilitation priming effects. By varying the relationship (e.g., physical, semantic, etc.) between the prime and the target, one can investigate the depth of nonconscious processing. However, masking has one considerable limitation: It cannot be used to create sustained periods of perceptual invisibility. Indeed, masking is effective only as long as stimulus duration does not exceed a few tens of milliseconds ( $\sim 50 \mathrm{~ms}$; see, e.g., Gelskov \& Kouider, 2010). Past evidence for restricted nonconscious influences might thus reflect methodological limits (i.e., short durations induce weak signals and, thus, weak influences), rather than theoretical constraints on the nature of nonconscious perceptual processes.

To overcome this practical limit, research has focused on alternative methods that offer longer periods of invisible 
stimulation, such as binocular rivalry (Tong, Meng, \& Blake, 2006). Rivalry reflects the fact that when two incompatible stimuli (e.g., a face and a house) are presented to an observer, one in each eye, they compete for perceptual awareness, which, as a result, oscillates between the two possible percepts. When one percept wins the competition, it is totally visible and then fully perceived by the observer, while the other percept is completely suppressed and, hence, rendered invisible for a few seconds. Nevertheless, perceptual switches in rivalry cannot be manipulated reliably (i.e., one cannot control the duration of invisible stimulation). Recently, an extension of rivalry termed continuous flash suppression (CFS) has been reported as a powerful method that can surmount both the limitations of visual masking and rivalry (Fang \& He, 2005; Tsuchiya \& Koch, 2005). In CFS, a series of contour-rich, high-contrast patterns are continuously flashed to one eye at $\sim 10 \mathrm{~Hz}$, profoundly and reliably suppressing salient visual information presented to the other eye (Tsuchiya, Koch, Gilroy, \& Blake, 2006). CFS offers an excellent control of timing (unlike rivalry) and can fully suppress an image presented for considerably long durations (unlike masking), thus potentially inducing stronger nonconscious influences on human behavior.

In the present study, we investigated this issue by studying the impact of sustained periods of invisibility and testing whether this indeed increases facilitatory nonconscious influences. To do so, we adapted a repetition face-priming paradigm previously used in the context of visual masking (Henson, Mouchlianitis, Matthews, \& Kouider, 2008; Kouider, Eger, Dolan, \& Henson, 2009) to the CFS method and tested whether invisible faces presented during a relatively long period might result in larger facilitation on subsequent target processing, in comparison with classical masked prime durations. Across six experiments, we showed that while relatively brief periods of subliminal stimulation $(60 \mathrm{~ms})$ result in classical facilitation effects, substantially longer periods $(1,000 \mathrm{~ms})$ actually lead to negative priming influences. Importantly, this overstimulation cost was specific to nonconscious perception, since conscious processing of the prime gave rise to facilitatory priming influences for both short and long durations. This nonconscious overstimulation cost reveals an important dissociation between conscious and nonconscious processing.

\section{Method}

Participants One hundred thirty-nine healthy right-handed university students from Paris (mean age, 23 years; range, 18-33) gave written informed consent to participate in the study for monetary compensation (22 in Experiment.1 18 in Experiment 2, 16 in Experiment 3, 42 in Experiment 4, 15 in Experiment 5, and 26 in Experiment 6). All participants were naïve as to the purpose of the experiment and had normal or corrected-to-normal vision.

Stimuli and apparatus Observers viewed the CRT display $(1,280 \times 1,024 ; 70 \mathrm{~Hz})$ through an adjustable mirror stereoscope at a distance of $45 \mathrm{~cm}$. A frame $\left(8^{\circ} \times 6^{\circ}\right)$, composed of black and white textured bars, was presented in each eye to facilitate binocular fusion. Each CFS pattern consisted of randomly generated forms of random colors and sizes. Face stimuli were grayscale pictures of a subset of 40 famous and 40 unknown faces (half male, half female) selected from a set used previously (de Gardelle, Charles, \& Kouider, 2011; de Gardelle \& Kouider, 2010). All images were matched for average luminance and shape to preclude overall stimulus shape as a confound. Importantly, to avoid retinotopic overlap with target faces, the primes were scaled to be smaller $\left(5.4^{\circ} \times 3.8^{\circ}\right)$ than the targets $\left(6.8^{\circ} \times 4.8^{\circ}\right)$.

Procedure The experimental design was typical of subliminal priming procedures. Observers first participated in an experimental session to measure priming influences, and then underwent an additional session to assess prime visibility. The general experimental paradigm is represented in Fig. 1. For each trial, a central cross was presented for $300 \mathrm{~ms}$. Then CFS patterns (dominant) were flashed in one eye at a frequency of $8 \mathrm{~Hz}$ over $1,125 \mathrm{~ms}$. The eye receiving the CFS patterns (left or right) was randomly determined for each trial. In the opposite eye, a prime stimulus (suppressed) was presented either during the last 1,000 ms (Fig. 1a), or during the last $60 \mathrm{~ms}$ of the CFS period (Fig. 1b). A monocular target was then presented for $700 \mathrm{~ms}$, either in the suppressed eye (same-eye condition) or in the dominant eye (different-eye condition; in Experiments 1-3 only). Each face stimulus belonged to either a famous person (e.g., pop star, politician, etc.) or an unknown person. Observers were asked to gaze at central fixation and pay attention during the flashes in order to respond as quickly as possible whether the upcoming face was famous or unknown. Targets were either a repetition of the prime (primed condition) or a different face from the same (Experiment 2 only) or the opposite (Experiments 1, 3-6) fame category (unprimed condition). Importantly, the size of the prime stimulus was reduced to avoid a low-level locus of repetition priming due to visual prime-target overlap (de Gardelle et al., 2011; de Gardelle \& Kouider, 2010; Kouider et al., 2009).

Prime visibility A prime visibility test (160 trials), in the form of a two-alternative forced choice discrimination task, was conducted immediately after the experimental session. Participants were informed about the presence of the first face 


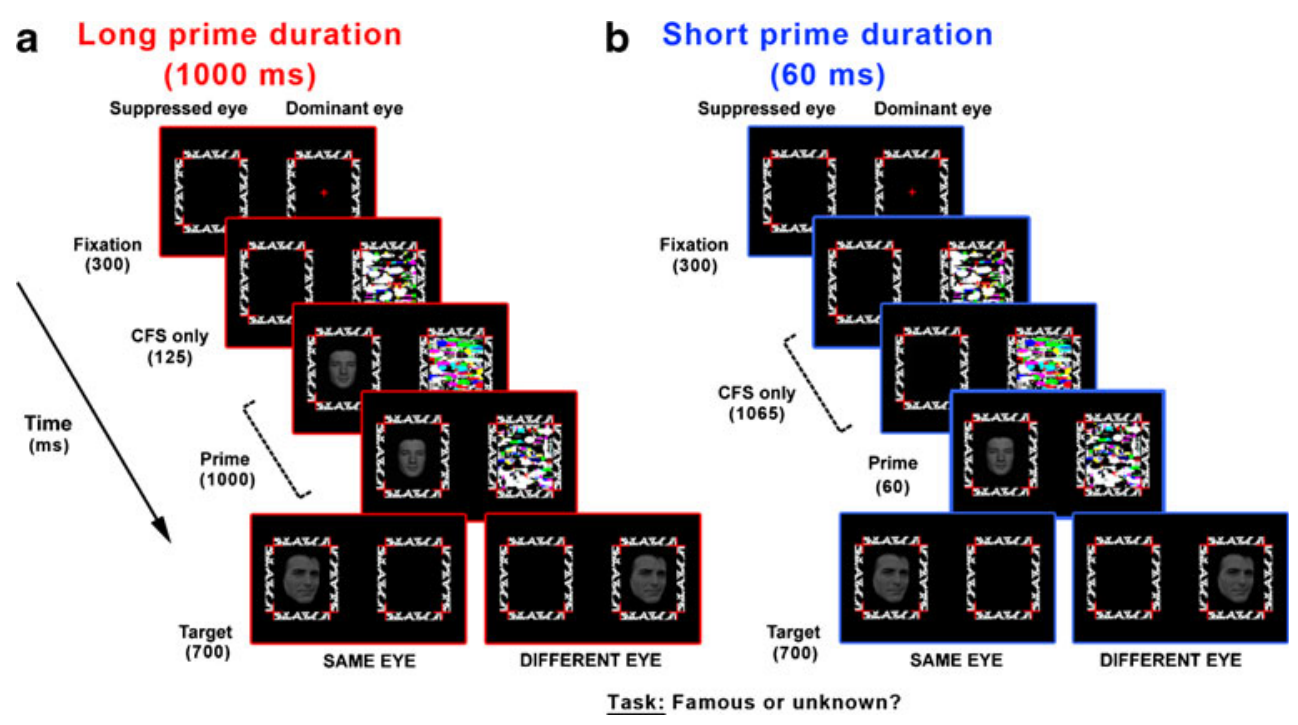

Fig. 1 Basic experimental design. CFS patterns were flashed in the dominant eye for $1,125 \mathrm{~ms}$ while a prime stimulus was presented in the opposite (suppressed) eye for either 1,000 ms (a; long prime presentation) or $60 \mathrm{~ms}$ (b; short prime presentation). In Experiment 5, no CFS flashes were presented during the suppression period, resulting in visible prime presentation. Targets were either a repetition of the prime (primed) or a different face (unprimed). Importantly, the size of the primes was reduced, as compared with target sizes, to avoid low-level prime-target interactions. Monocular target and prime stimuli were presented either in the same eye or in different eyes during the CFS period. The procedure was identical to that in the experimental priming session, with the exception that instead of the target face, two different faces were presented: one corresponding to the prime, and the other to a different face from the same (Experiment 2 only) or the opposite (Experiments 1,3-6) fame category. The prime was equally likely to appear on the left or right side. Observers were asked to indicate which one of these two faces was presented during the CFS period and were told that only accuracy, not response time (RT), was important. Performance on the prime visibility task was analyzed offline, and only the data of participants whose performance in the prime visibility test was below $60 \%$ were used in further analysis.

Analysis For the six experiments, median RTs were calculated for each observer after exclusion of errors and trials with RTs below $200 \mathrm{~ms}$ and above 1,400 $\mathrm{ms}$ (fewer than $5 \%$ of the trials were excluded in each experiment). The means of the individual median RTs are reported in subsequent analyses as mean average RTs. An omnibus, repeated measures ANOVA was followed by more focused $2 \times 2$ ANOVAs. Planned pairwise tests were used to assess reliability of the effects. All $t$-tests were two-tailed. Pearson's correlation coefficient was used for correlation tests.

\section{Results}

Three different factors were manipulated: priming (primed vs. unprimed), familiarity (famous vs. unknown), and prime-target ocular relation (same vs. different eye). However, since data for the different-eye condition did not reveal any priming effects in any of the experiments that included the different-eye condition (Experiments 1-3), we focus below on the analysis of the same-eye condition. Results of the different-eye condition are presented in the Supplemental Material (see Fig. S1) and will be addressed in the Discussion section. RTs for the same-eye condition are presented in Fig. 2 (see also the Supplemental Material, Table S1, for a more detailed description of the results).

Prime visibility Across 139 participants, 104 met the specified criteria for inclusion corresponding to performance in the prime visibility test below 60\% (104/139 across six experiments: Experiment 1, 18/22; Experiment 2, 16/18; Experiment 3, 16/16; Experiment 4, 24/42; Experiment 5, 15/15; and Experiment 6, 15/26). Global accuracy and $d$ values for the prime visibility test are reported in Table 1 . The $d$ values were not significantly different from zero $(p>.1)$, confirming that participants included in the following analysis of priming were indeed unaware of the suppressed prime stimuli.

Priming The goal of Experiment 1 was to investigate the existence of a repetition priming effect induced by long suppressed $(1,000 \mathrm{~ms})$, rather than brief masked, faces. While previous CFS studies have reported weak, but still measurable, stimulus-induced neural activity for suppressed faces (Jiang \& He, 2006; Sterzer, Haynes, \& Rees, 2008; Sterzer, Jalkanen, \& Rees, 2009), the possibility of face 
Fig. 2 Behavioral priming results: Mean response times (RTs) for Experiment 1 (a), 2 (b), and 3 (c) in the same-eye condition (for data in the different-eye condition, see Supplementary Material, Fig. S1). $* * * p<.001$, $* * p<.01,{ }^{*} p<.05$. Error bars represent SEMs for RTs across participants

\section{a}

Expt. 1

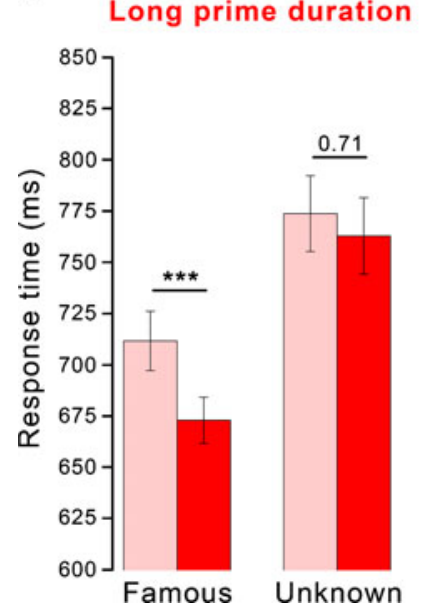

b

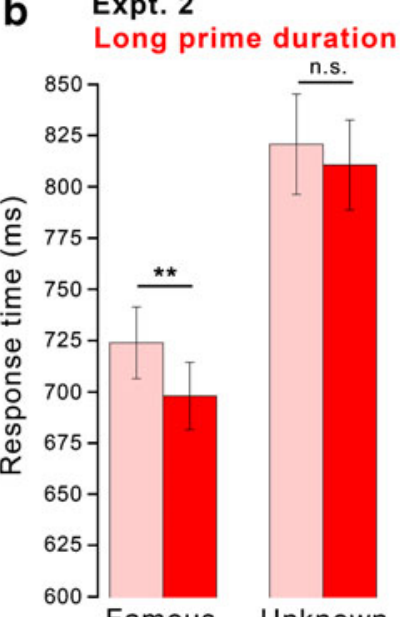

Famous c

Expt. 3

Short prime duration
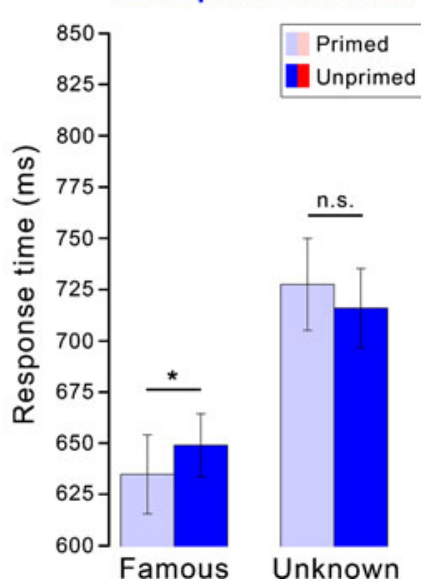

repetition priming influences on subsequent processing has not been tested so far. A $2 \times 2$ ANOVA (primed vs. unprimed $\times$ famous vs. unknown) showed main effects of familiarity, $F(1,17)=62, p<.001$, and of priming, $F(1,17)=18.5, p<.001$, with a reliable familiarity $\times$ priming interaction, $F(1,17)=12.3, p<.005$. As is illustrated in Fig. 2a, priming influences were restricted to famous faces (unknown: $p=.071$ ). Surprisingly, this effect was not associated with shorter RTs but with longer RTs in the primed condition, indicating that the subliminal prime stimulation actually impaired, rather than facilitated, the processing of the target $(M=-38.7 \mathrm{~ms}), t(17)=4.7$, $p<.001$. The observation of priming restricted to famous faces here is consistent with the masked priming literature (Kouider \& Dehaene, 2007; Kouider et al., 2009) and supports the idea that familiar information enjoys an advantage when processed under conditions of interocular suppression (Jiang, Costello, \& He, 2007). We return to this idea in the Discussion section.

In Experiment 2, we explored whether the counterintuitive negative priming observed in Experiment 1 resulted from prime-target interactions at the perceptual or, rather, at the motor response locus. Indeed, past studies in the

masking literature have shown that negative priming can be the result of an automatic inhibitory mechanism that suppresses the response initiation induced by the prime (Eimer \& Schlaghecken, 2003; Sumner, Tsai, Yu, \& Nachev, 2006). In these studies, overlearned stimulusresponse associations (e.g., left-hand responses for leftarrow stimuli) can be induced even for masked and brief stimuli, resulting in facilitatory priming when the prime and target are congruent (i.e., both corresponding to the same motor response). However, when the prime-target interval is increased, the partial motor activation initially induced by the prime is inhibited, resulting in negative priming (note that in these experiments, only the interval duration can be increased, while prime presentation can only be brief, due to the intrinsic limitations of masking described above). Since in Experiment 1, unrelated prime-target pairs were also from the opposite fame category (i.e., leading to the opposite motor response), it is possible that our finding reflects visuo-motor inhibition of the response associated to the prime. In Experiment 2, we specifically tested this hypothesis by using prime-target pairs belonging to the same category (i.e., associated with the same motor response) in both primed and unprimed conditions. For

Table 1 Average percent correct and $d^{\prime}( \pm S E M)$ for the two-alternative forced choice prime visibility tests

\begin{tabular}{|c|c|c|c|c|c|c|}
\hline & \multirow{2}{*}{$\begin{array}{l}\text { Expt. } 1 \\
\text { Long prime } \\
\text { duration }\end{array}$} & \multirow{2}{*}{$\begin{array}{l}\text { Expt. } 2 \\
\text { Long prime } \\
\text { duration }\end{array}$} & \multirow{2}{*}{$\begin{array}{l}\text { Expt. } 3 \\
\text { Short prime } \\
\text { duration }\end{array}$} & \multicolumn{2}{|l|}{ Expt. 4} & \multirow{2}{*}{$\begin{array}{l}\text { Expt. } 6 \\
\text { Short prime } \\
\text { duration/ Long SOA }\end{array}$} \\
\hline & & & & $\begin{array}{l}\text { Short prime } \\
\text { duration }\end{array}$ & $\begin{array}{l}\text { Long prime } \\
\text { duration }\end{array}$ & \\
\hline \multicolumn{7}{|l|}{ Famous } \\
\hline - accuracy $(\%) \pm$ SEM & $50.3 \pm 2$ & $49 \pm 2.4$ & $48.5 \pm 1.7$ & $49.3 \pm 6.9$ & $46 \pm 2.2$ & $51 \pm 1.5$ \\
\hline - d' \pm SEM & $0.01 \pm 0.07$ & $-0.04 \pm 0.08$ & $-0.04 \pm 0.06$ & $-0.04 \pm 0.36$ & $-0.14 \pm 0.08$ & $0.04 \pm 0.05$ \\
\hline \multicolumn{7}{|l|}{ Unknown } \\
\hline - accuracy $(\%) \pm$ SEM & $51 \pm 2.3$ & $51 \pm 2.5$ & $52 \pm 2$ & $50.2 \pm 5.5$ & $56 \pm 2.4$ & $53 \pm 2.4$ \\
\hline - d' \pm SEM & $0.04 \pm 0.08$ & $0.05 \pm 0.1$ & $0.06 \pm 0.07$ & $-0.02 \pm 0.3$ & $0.26 \pm 0.1$ & $0.12 \pm 0.09$ \\
\hline
\end{tabular}


instance, in the unprimed condition, the prime and the target corresponded to different faces but belonged to the same fame category and, thus, to the same response category. Any priming effect could not be attributed to priming of specific motor response (Abrams \& Greenwald, 2000; Damian, 2001; Kouider \& Dupoux, 2007). As is presented in Fig. 2b, we found a main effect of priming, $F(1$, $15)=5.4, p<.05$, which again reflected reliable negative priming restricted to famous faces [famous, $M=-26 \mathrm{~ms}, t$ $(15)=2.9, p<.01$; unknown, $p>.1]$, revealing that negative priming influences result from prime-target interactions at perceptual, rather than motor, levels.

In Experiment 3, we questioned whether the negative priming found in Experiments 1 and 2 resulted specifically from reliance on interocular suppression or, rather, from sustained periods of nonconscious stimulation. In the former case, priming would remain negative whatever the prime duration. In the later case, negative priming would result only from the relatively long presentation of the prime $(1,000 \mathrm{~ms})$, as compared with the brief presentations traditionally used in masked priming experiments. In order to address this issue, we used exactly the same procedure as in Experiment 1, with the only exception that the prime duration was now reduced to $60 \mathrm{~ms}$. Interestingly, as is depicted in Fig. $2 c$, we found that the priming effect now turned into a positive effect [famous, $M=+14.2 \mathrm{~ms}, t(15)=2.34, p<$ .05 ; unknown, $p>.1$ ]. Thus, the negative priming effects observed in the previous experiments are directly related to the use of long periods of nonconscious prime stimulation.

Experiment 4 was aimed at confirming the detrimental effect of subliminal overstimulation on subsequent processing, by relying on a within-subjects design with long $(1,000 \mathrm{~ms}$, as in Experiment 1$)$ versus short $(60 \mathrm{~ms}$, as in Experiment 3) prime durations. Prime duration conditions were blocked and the order was counterbalanced across observers (long followed by short and vice versa). From here, we restricted prime-target presentation to the same eye only. Importantly, this design also allowed us to examine the relationship between facilitatory and inhibitory effects on a participant-by-participant basis. Indeed, one possibility is that priming effects reflect the expression of two independent processes: a fast and transient facilitatory component, stronger at early phases of processing, and an inhibitory component that grows over time, resulting in a transition from positive to negative priming with increased stimulation duration. In this case, one would expect facilitatory and inhibitory effects to be additive and correlate on a participant-by-participant basis. Observers with stronger facilitation at short durations would show weaker inhibition at long durations. An alternative explanation is that there are two mechanisms that relate to each other such that stronger facilitation at early phases of processing intensifies the buildup of the inhibition component, resulting in stronger inhibition at long durations. In this case, one would expect that positive and negative priming effects anticorrelate: the stronger the facilitation at short durations, the stronger the inhibition at long durations.

We largely replicated both the positive and the negative priming effects for short and long prime presentations, respectively (Fig. 3a). A $3 \times 3$ ANOVA revealed a robust interaction between priming and prime duration, $F(1,23)=$ $19.1, p<.001$, as well as an interaction between familiarity, priming, and prime duration, $F(1,23)=10.7, p<.005$. For the short prime duration, we found that the main effect of priming, $F(1,23)=5.2, p<.05$, reflected positive priming [famous, $M=+19.4 \mathrm{~ms}, t(23)=2.5 p<.05$; unknown, $p>.1]$. For the long prime duration, the main effect of priming, $F(1,23)=25.7, p<.001$, reflected negative priming [famous, $M=-40.3 \mathrm{~ms}, t(23)=5.4, p<.001$; unknown, $p>.1]$. Note that we observed similar RTs for the unprimed condition for both short and long prime presentations, indicating that the prime specifically alters the processing of the target for repetitions. Furthermore, we observed a significant anticorrelation between the magnitude of individual priming at short and long prime durations (Fig. 3b), $r=-.44, t(23)=2.3, p<.05$. That is, given the same observer, stronger positive priming in the short prime duration condition was associated with stronger negative priming in the long prime duration condition, indicating that facilitatory and inhibitory processes are not independently expressed as a function of stimulation duration.

In Experiment 5, we conducted a supraliminal version of Experiment 4 to investigate the impact of perceptual awareness on the directionality of priming. Specifically, we were interested in whether the cost that results from relatively long periods of prime stimulation depends on whether the prime is consciously or nonconsciously perceived. The design was the same as in Experiment 4, except that no CFS flashes were presented, allowing the prime to be clearly visible under those conditions. A $3 \times 3$ ANOVA revealed a robust interaction between priming and prime duration, $F(1,14)=15.6$, $p<.005$, and between familiarity and priming, $F(1,14)=$ $15.6, p<.005$, as well as an interaction between familiarity, priming, and prime duration, $F(1,14)=18.7, p<.001$. As in the previous experiments, we found (Fig. $3 \mathrm{c}$ ) that the brief presentation of the prime resulted in a positive priming effect [famous: $M=+51 \mathrm{~ms}, t(14)=4.64, p<.001]$. More important, the relatively long presentation of the prime was now associated with a large positive priming effect [famous: $M=+171 \mathrm{~ms}, t(14)=8.15, p<.001]$. In addition, priming effects were also observed for unknown faces [short duration, $M=+31 \mathrm{~ms}, t(14)=4.1, p<.005$; long duration, $M=+58 \mathrm{~ms}, t(14)=3.93, p<.005]$. These results suggest important qualitative differences in the processing of visual 


\section{a}

Expt. 4 - Invisible prime

Short prime duration

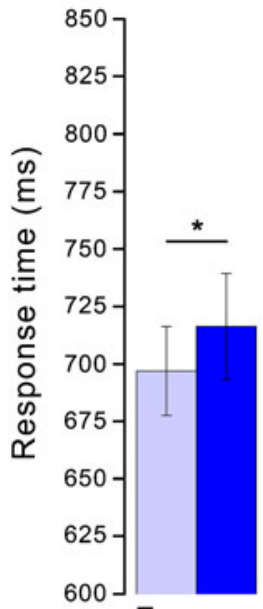

Famous
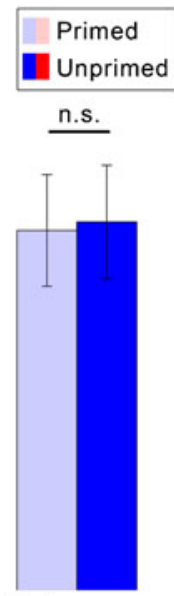

Unknown

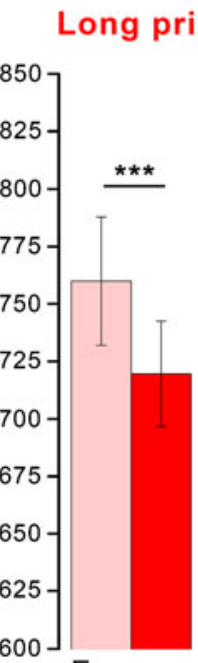

Famous

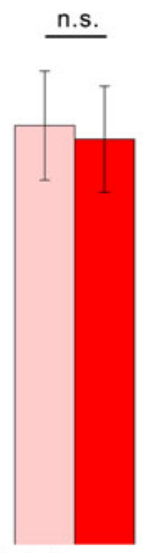

Unknown

\section{b}

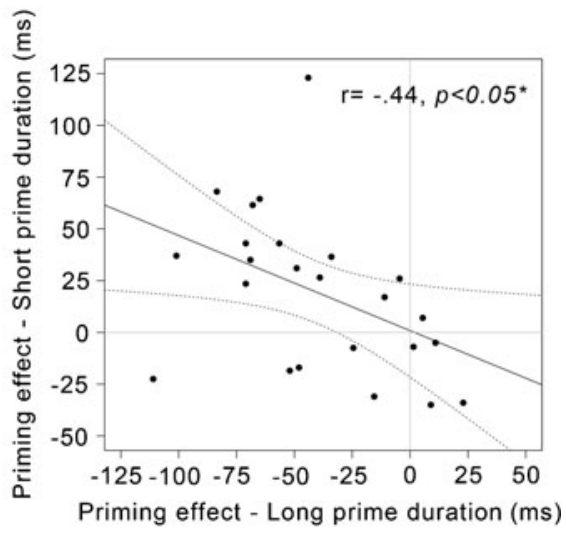

d

Fig. 3 Behavioral priming results: Mean response times (RTs) for Experiment 4 (a; invisible prime) and Experiment 5 (c; visible prime) are each presented for short and long prime durations. b Correlation of individual's famous face priming effects for long and short invisible

information in the presence or absence of awareness (Fig. 3d).

One could argue, however, that because prime stimuli were made visible by removing the CFS flashes, the dissociation between conscious and nonconscious processing was due not to prime visibility per se, but to the presence or absence of interocular competition. To confirm the crucial role of prime visibility, we conducted an additional analysis of the data from Experiment 4, including participants previously rejected on the basis of their performance on the prime visibility test (see the Method section). Interestingly, for the long prime condition, we found a positive correlation between priming and prime visibility, with individual priming effects going from negative to positive with prime visibility (Fig. $4 \mathrm{a} ; r=.60, p<.001 ; y$ -
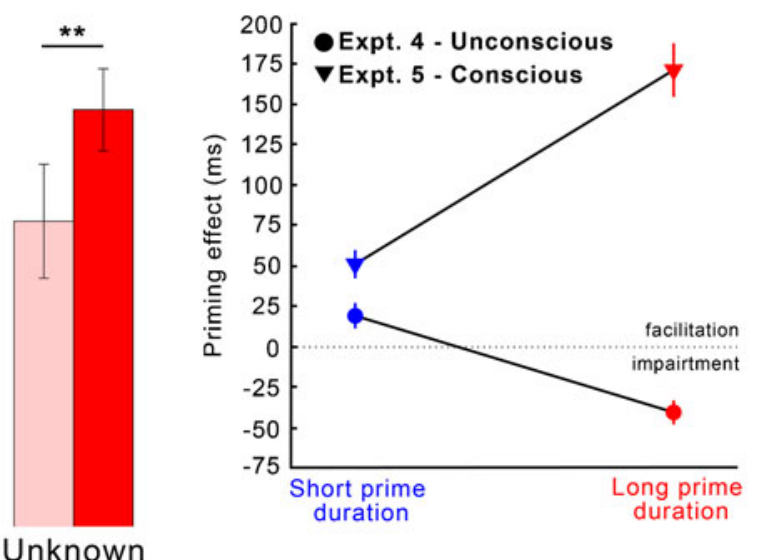

prime durations in Experiment 4. d Priming effects for famous faces as a function of prime duration (short vs. long) and prime visibility (invisible, Experiment, 4 vs. visible, Experiment 5). ***p<.001, ** $p<.01,{ }^{*} p<.05$. Error bars represent SEMs for RTs across participants

intercept $=-30 \mathrm{~ms}, p<.001)$. No correlation between priming and prime visibility was observed in the short prime condition (Fig. 4a; $r=-.17 \mathrm{~ms}, p=.26 ; y$-intercept $=+14.8 \mathrm{~ms}$, $p<.001)$. Interestingly, an analysis restricted to observers able to perceive the prime in the long prime duration condition (performance above $70 \%$ in the prime visibility test) revealed a positive priming effect [Fig. $4 \mathrm{~b}$; famous, $M=+28 \mathrm{~ms}, t(1,7)=$ 2.5, $p<.05$; unknown, $p>.1]$. Altogether, our findings support a crucial role of prime visibility in the directionality of priming patterns, providing strong evidence that processing a stimulus with or without perceptual awareness can result in qualitatively different behavioral consequences.

Finally, in Experiment 6, we questioned whether the direction of priming was related to the duration of prime 
Fig. 4 a Correlation between individual's famous face priming effects and individual's prime visibility for short and long prime presentations in Experiment 4 , including all the participants tested $(n=42)$. b

Behavioral priming results: Mean response times (RTs) for Experiment 4 , including high prime visibility $(d)$ participants $(n=8)$. $* * * p<.001, * * p<.01,{ }^{*} p<.05$. Error bars represent SEMS for RTs across participants a

Expt. 4 - All Observers $(n=42)$

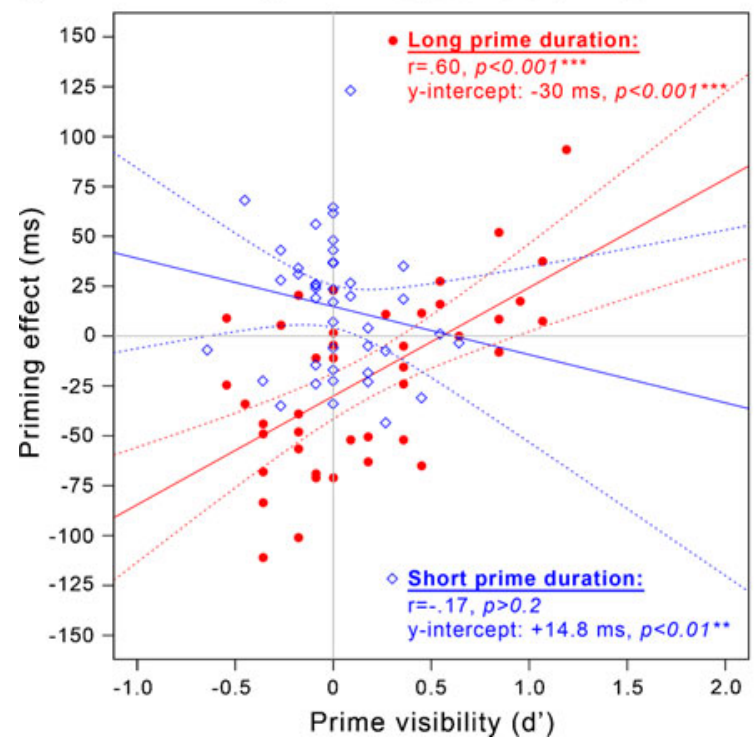

b

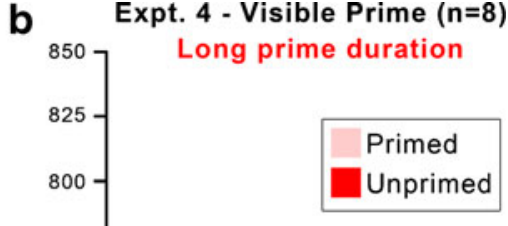

stimulation per se or to the stimulus onset asynchrony (SOA) between the prime and the target. Prime stimuli appeared 1,000 ms before the target (i.e., SOA similar to the long stimulation condition) but were presented for $60 \mathrm{~ms}$ (i.e., prime duration similar to the short stimulation condition). If the SOA determines the directionality of priming, we expected to observe negative priming influences. In contrast, if the direction of priming is determined by the prime duration, positive priming influences should be observed. As is shown in Fig. 5, we found an interaction between familiarity and priming, $F(1,14)=7.9, p<.05$, reflecting a positive priming effect that was restricted to famous faces [famous, $M=+11 \mathrm{~ms}, t(14)=2.4, p<.05$; unknown, $p>.1]$. This finding suggests that the duration of prime stimulation determines the pattern of priming observed.

\section{Discussion}

By combining two powerful approaches, the masked priming paradigm and the CFS method, we investigated whether long periods of invisible prime stimulation result in larger nonconscious influences than with the brief prime durations usually employed in masking studies. While brief presentations of invisible primes resulted in classical facilitation effects, long periods led to negative priming effects. Interestingly, for relatively long periods of prime stimulation, we found a relationship between prime visibility (invisible vs. visible) and the directionality of priming (negative vs. positive), going from negative to positive influences with increasing levels of prime visibility. Taken together, our results reveal that stimulus processing in the presence or absence of perceptual awareness can lead to qualitatively different behavioral consequences.

Increased invisible stimulation reverses priming influences While brief masked prime stimuli usually facilitate subsequent processing, some studies have reported evidence of negative priming effects, reflecting costs on compatible trials (Eimer \& Schlaghecken, 2003; Sumner et al., 2006). The interval between the prime and the target has been

\section{Expt. 6}

\section{Short prime duration I Long SOA}

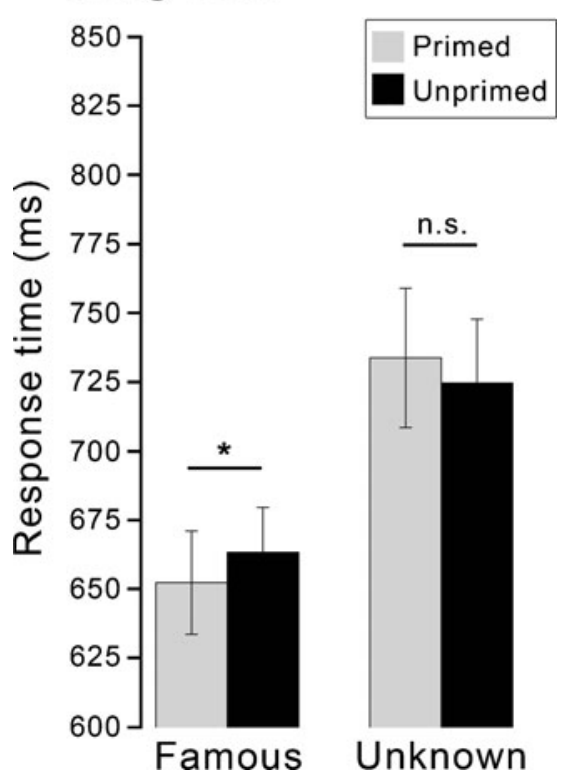

Fig. 5 Behavioral priming results: Mean response times (RTs) for Experiment $6 .{ }^{* * *} p<.001,{ }^{* *} p<.01,{ }^{*} p<.05$. Error bars represent SEMs for RTs across participants 
suggested to be determinant in the transition from positive to negative priming (Sumner, 2007). With short primetarget intervals $(<100 \mathrm{~ms})$, facilitation occurs on compatible trials, but when the interval becomes longer, negative priming effects are observed. Our results suggest, however, that the duration of invisible prime stimulation, rather than the SOA between the prime and the target, is what determines the pattern of priming observed. Wentura and Frings (2005) reported a similar pattern of priming, using a different methodology. In their paradigm, a masked prime word was repeatedly flashed. By varying the number of repetitions of the masked prime, they were able to present the primes nonconsciouly for as long as a second. Similar to our findings, several repetitions of the masked prime led to negative priming influences. An important distinction from our study is that, in their paradigm, the masked primes were not continuously presented. Therefore, it was unclear whether the negative priming effects were the consequence of longer invisible prime stimulation or just the effects of prime repetition. The use of the CFS technique allowed us to identify the prime stimulation duration as the key determinant of the transition from positive to negative nonconscious repetition priming.

Negative effects were originally interpreted as resulting from automatic self-inhibition mechanisms that suppress the initial prime-induced response activation (Eimer \& Schlaghecken, 2003). The persistence of our effect using congruent prime-target pairs (Experiment 2) rejects an interpretation in terms of motor response inhibition and suggests that the transition from positive to negative priming with prime duration reflects perceptual interactions between the prime and the target. An alternative theory, the object-updating account (Lleras \& Enns, 2004, 2005, 2006), has been proposed to take into account both motor and nonmotor negative priming evidence. According to this theory, perception reflects an update of what is new in the display. Long periods of prime stimulation make subsequent targets that are similar to the prime less salient, leading to negative priming effects.

In line with the object-updating theory, Huber and colleagues (Huber \& O'Reilly, 2003; Huber, Shiffrin, Lyle, $\&$ Ruys, 2001) have developed a neural habituation priming model to explain the change from positive to negative priming with increasing prime duration. This model is based on the idea that perception emphasizes new against old information. Representations associated with the prime are quickly activated at prime onset but become less relevant with time, due to inhibitory mechanisms that automatically reduce the influence of recent information over time. Thus, while repeated targets presented after brief stimulation periods are facilitated by residual prime-onset activation, longer periods of stimulation impair subsequent recognition. A considerable advantage of this model is that it is based on neurophysiological mechanisms that are well understood. Single-unit recordings have shown that prime onset induces a fast peak activation of the neurons representing prime information that can give a head start to subsequent processing. However, following the rapid onset activation, neural activity falls off to a low asymptotic level despite constant stimulation. This rise-and-fall activation pattern has been proposed to result from short-term habituation mechanisms that correspond to the progressive depletion of synaptic resources with sustained stimulation (Abbott, Varela, Sen, \& Nelson, 1997). While reflecting a cost (negative priming) under certain circumstances, habituation mechanisms are generally beneficial for our perception, serving to reduce source confusion from recent stimulations (Huber, 2008).

Although this model has been developed to explain priming effects obtained using clearly visible primes, similar mechanisms could explain the change from positive to negative priming with subliminal stimulation. Indeed, neural habituation is ubiquitous in neural processing and is considered to be an automatic mechanism triggered by the prior activity of the neuron (Abbott et al., 1997; Huber \& O'Reilly, 2003). Thus, activations induced by subliminal stimulation should result in early peak onset responses that are attenuated via habituation, resulting in a transition from positive to negative priming with increasing stimulation duration.

Our findings mirror a number of examples where processing is inhibited by prior stimuli presented for long durations but is facilitated by brief ones. For instance, previewing one half-image of a binocular rivalry pair generally results in its suppression when the other half is added, a classical phenomenon called flash suppression (Wolfe, 1984). A recent study (Brascamp, Knapen, Kanai, van Ee, \& van den Berg, 2007) showed that under certain circumstances, previewing an image can facilitate its initial dominance upon rivalry presentation, a phenomenon called flash facilitation. Interestingly, they demonstrated that previewing an image will lead to facilitation or suppression depending on the duration of the prior stimulus. Similar to our results, they found that brief prior stimuli facilitate, whereas long prior stimuli suppress. Such antagonism between long and short prior stimulation on subsequent perception might be a general feature. For instance, perceptual history has been shown to influence subsequent ambiguous perception on different time scales (Brascamp et al., 2008; Kornmeier, Hein, \& Bach, 2009; Pearson \& Brascamp, 2008).

Increased invisible stimulation reveals a dissociation between conscious and nonconscious processing An ongoing debate in the negative priming literature concerns the causal role of prime visibility in the directionality of priming (Sumner, 2007). Although changes from positive 
to negative priming are not always related to prime visibility, many studies have reported a relationship between the level of prime visibility and the direction of priming, with awareness generally moderating negative priming (Eimer \& Schlaghecken, 2003; Frings \& Wentura, 2005; Sumner et al., 2006). Our data support a causal role of prime visibility in the transition from positive to negative priming. Indeed, while brief periods of prime stimulation resulted in positive priming effects regardless of prime awareness, long stimulation periods revealed qualitatively different effects for visible (facilitation) and invisible (impairment) prime stimuli. Perceptual awareness of the prime might lead to stronger and sustained activation that overcomes the inhibition processes and continues to facilitate subsequent perception for longer periods than with invisible primes. A recent study that investigated conscious repetition face priming while manipulating prime duration closely parallels our results (Rieth \& Huber, 2010). In this study, brief prime durations produced positive priming, whereas long prime durations led to negative influences. This finding suggests that an overstimulation cost should have also been observed with visible primes (Experiment 5), using longer prime durations. Thus, visual awareness seems to delay the transition from positive to negative priming. Although short-term habituation is considered to be automatic, flexible strategic modulations associated with conscious prime representations might result in qualitatively different neural dynamics. Habituation occurs at many levels of perceptual representation, with lower levels of processing activated and habituated more quickly than higher levels of representation (Carandini, Heeger, \& Senn, 2002; Huber \& O'Reilly, 2003; Huber et al., 2001).

The locus of repetition priming under interocular suppression One might argue that our results reflect lowlevel visual, rather than face-specific, repetition priming. Yet prime stimuli were all reduced in size to avoid retinotopic overlap with target stimuli, ruling out low-level explanations. Indeed, scale-invariant interactions between face stimuli have been reported to reflect interactions at the level of face-selective areas (Eger, Schyns, \& Kleinschmidt, 2004), such as the fusiform face area (FFA; Kanwisher \& Yovel, 2006). More important, we found that only famous faces elicit nonconscious priming. This aspect of our results suggests that nonconscious priming influences arises only when the invisible prime triggers some preexisting target face representations. Alternatively, the absence of a priming effect with unknown faces might be due to the fame judgment task we used. In the primed condition (in which the target is the same as the prime), the preexposure to the unknown prime might have induced a feeling of familiarity of the target that would have interfered with the fame judgment task (Jacoby, Kelley, Brown, \& Jasechko, 1989).
While it is largely accepted that lower levels of processing (e.g., motor response, low-level features, etc.) do not require awareness, the existence of high-level processing along the ventral stream (e.g., categoryspecific, semantic processing, etc.) under interocular suppression is currently subject to debate (Lin \& He, 2009; Tong et al., 2006). For instance, Fang and He (2005) reported category-specific responses for invisible manipulable tool-like objects in dorsal cortical areas, but not for invisible faces that are represented in ventral areas. Following up on this observation, a recent CFS study (Almeida, Mahon, Nakayama, \& Caramazza, 2008) showed category-specific priming for invisible tools, but not for other categories (i.e., vehicles and animals) that involve ventral regions. However, recent studies have shown that residual information might also reach high-level stages of the ventral stream. For instance, responses to invisible neutral faces, as compared with visual patterns matched for low-level similarity, were still measurable in the FFA, albeit much reduced under CFS (Jiang \& He, 2006). There is also evidence of category-specific processing of suppressed faces in the FFA, using more sensitive analysis methods based on multivariate pattern classification (Sterzer et al., 2008). In addition, adaptation to suppressed face stimuli appears to strongly depend on spatial attention (Shin, Stolte, \& Chong, 2009; Yang, Hong, \& Blake, 2010).

On the one hand, the repetition priming effects observed in our study might not be face specific but, rather, the consequences of size-invariant repetition priming (Eddy \& Holcomb, 2009; Vuilleumier, Henson, Driver, \& Dolan, 2002). On the other hand, it is possible that our effects are face specific, since residual information seems to be able to reach face-specific regions despite interocular suppression. Again, the specificity of our effects to famous faces suggests that preexisting face representations are necessary but could also be explained by the fame judgment task used. Further work (e.g., using neuroimaging methods) is needed in order to determine whether the repetition priming effects observed in the present study are specific to faces.

Another important aspect of our results regards the restriction of subliminal influences to prime-target stimuli presented in the same eye. Such a high degree of ocular specificity might reflect an early locus of priming (i.e., monocular neurons in LGN and V1). However, if priming influences were indeed the consequence of low-level interactions at monocular stages, repetition effects should have been observed whatever the fame category was, which was not the case here. In addition, the RTs were globally faster in the different-eye condition than in the same-eye condition, regardless of the priming condition. This cost might be due to reduced sensitivity to the monocular target in the previously suppressed eye (i.e., same-eye condition), as compared with the previously dominant eye (i.e., 
different-eye condition). Sensitivity to monocular test stimulus has been shown to be reduced when presented to the eye that was previously suppressed (Freeman \& Li, 2009). Such differences in sensitivity between the suppressed and the dominant eyes might have interfered with the nonconscious priming influences in the different-eye condition. Note that previous CFS studies have generally presented stimuli either in both eyes or in the same eye as the suppressed stimuli. Therefore, previous subliminal findings might have also been restricted to the suppressed eye. For instance, a recent CFS study has reported reward reinforcement learning of invisible stimuli restricted to the suppressed eye - that is, without transfer across eyes (Seitz, Kim, \& Watanabe, 2009). In sum, the high degree of ocular specificity observed in our study (and potentially in others) could be intrinsic to the use of interocular suppression methods.

Finally, we should stress that the nonconscious overstimulation cost reported in this study is, by contrast, not specific to the use of interocular suppression methods. Indeed, in parallel to the present study, we used a novel method called gaze-contingent crowding (Faivre \& Kouider, 2011; Kouider, Berthet, \& Faivre, 2011) to similarly address the question of how stimulation duration shapes priming and habituation effects as a function of awareness (Faivre \& Kouider, in press). In this study, participants had to classify the orientation of a tilted grating (i.e., a Gabor patch) presented in the periphery. This target stimulus was preceded by another oriented grating that served as a prime and for which the orientation could be rendered nonconscious (i.e., nondiscriminable) by the joint use of crowding (i.e., the prime stimulus was surrounded by flankers) and gaze-contingent control through highresolution eye tracking (i.e., the prime stimulus was removed whenever the participant attempted to stare at it). Priming was measured by comparing prime-target pairs with the same orientation versus prime-target pairs with different orientations, while the prime orientation was either discriminable or nondiscriminable. As in the present study, we found a nonconscious overstimulation cost with positive priming when stimulating for relatively short durations (i.e., $200 \mathrm{~ms}$ ) and, conversely, negative priming when stimulating for longer durations (i.e., 1,000 ms). In addition, this biphasic pattern was observed even when the prime and target stimuli were presented in contrast counterphase, ruling out a purely low-level interpretation. It is of note, however, that the impact of stimulus awareness differed across the two studies. While conscious perception of the prime in the present CFS study led to a full reversal of the priming pattern, with now a large facilitation for visible long-lasting faces (see Fig. 4d), rendering the long-lasting gratings visible also canceled the negative effect, but priming remained weaker than with short visible stimuli (see Faivre \& Kouider, in press, for futher discussions on how stimulus awareness delays adaptation and transforms rise-and-reverse priming into rise-and-fall positive priming patterns). Although the exact mechanisms underlying the interaction between stimulation duration and perceptual awareness remain to be elucidated, both studies converge in demonstrating an overstimulation cost under conditions of invisibility.

\section{Conclusion}

By comparing brief versus long periods of subliminal stimulation, we brought to light a surprising biphasic pattern of priming: While brief processing of invisible information facilitates subsequent processing, longer subliminal stimulation actually impairs it, leading to what we have termed a nonconscious overstimulation cost. Such transition from positive to negative priming with increased stimulation duration might be explained in terms of habituation, a ubiquitous mechanism used to parse perceptual events and avoid source confusion. Overall, our results reveal that processing with or without perceptual awareness can lead to qualitatively different consequences with increasing stimulation duration.

Acknowledgments The authors thank Jeremy Wolfe, Nao Tsuchyia, David Huber, and two anonymous reviewers for their useful comments, as well as Inga Vendelin and Anne-Caroline Fievet for their assistance in data collection. This work was supported by an REI (Recherche Exploratoire et Innovation) grant from the French government.

\section{References}

Abbott, L. F., Varela, J. A., Sen, K., \& Nelson, S. B. (1997). Synaptic depression and cortical gain control. Science, 275, 220-224.

Abrams, R. L., \& Greenwald, A. G. (2000). Parts outweigh the whole (word) in unconscious analysis of meaning. Psychological Science, 11, 118-124.

Almeida, J., Mahon, B. Z., Nakayama, K., \& Caramazza, A. (2008). Unconscious processing dissociates along categorical lines. Proceedings of the National Academy of Sciences, 105, 15214 15218.

Brascamp, J. W., Knapen, T. H., Kanai, R., Noest, A. J., van Ee, R., \& van den Berg, A. V. (2008). Multi-timescale perceptual history resolves visual ambiguity. PLoS One, 3, e1497.

Brascamp, J. W., Knapen, T. H., Kanai, R., van Ee, R., \& van den Berg, A. V. (2007). Flash suppression and flash facilitation in binocular rivalry. Journal of Vision, 7, 11-12.

Carandini, M., Heeger, D. J., \& Senn, W. (2002). A synaptic explanation of suppression in visual cortex. Journal of Neuroscience, 22, 10053-10065.

Damian, M. F. (2001). Congruity effects evoked by subliminally presented primes: Automaticity rather than semantic processing. Journal of Experimental Psychology: Human Perception and Performance, 27, 154-165. 
de Gardelle, V., Charles, L., \& Kouider, S. (2011). Perceptual awareness and categorical representation of faces: Evidence from masked priming. Consciousness and Cognition. Advance online publication. doi:10.1016/j.concog.2011.02.001

de Gardelle, V., \& Kouider, S. (2010). How spatial frequencies and visual awareness interact during face processing. Psychological Science, 21, 58-66.

Eddy, M. D., \& Holcomb, P. J. (2009). Electrophysiological evidence for size invariance in masked picture repetition priming. Brain and Cognition, 71, 397-409.

Eger, E., Schyns, P. G., \& Kleinschmidt, A. (2004). Scale invariant adaptation in fusiform face-responsive regions. NeuroImage, 22, 232-242.

Eimer, M., \& Schlaghecken, F. (2003). Response facilitation and inhibition in subliminal priming. Biological Psychology, 64, 7-26.

Faivre, N., \& Kouider, S. (2011). Multi-feature objects elicit nonconscious priming despite crowding. Journal of Vision, 11(3, Art. 2), 1-10.

Faivre, N., \& Kouider, S. (in press). Increased sensory evidence reverses nonconscious priming during crowding. Journal of Vision.

Fang, F., \& He, S. (2005). Cortical responses to invisible objects in the human dorsal and ventral pathways. Nature Neuroscience, 8, $1380-1385$.

Freeman, A. W., \& Li, D. F. (2009). Conditions required for binocular rivalry suppression. Attention, Perception, \& Psychophysics, 71, 174-182.

Frings, C., \& Wentura, D. (2005). Negative priming with masked distractor-only prime trials: Awareness moderates negative priming. Experimental Psychology, 52, 131-139.

Gelskov, S. V., \& Kouider, S. (2010). Psychophysical thresholds of face visibility during infancy. Cognition, 114, 285-292.

Henson, R. N., Mouchlianitis, E., Matthews, W. J., \& Kouider, S. (2008). Electrophysiological correlates of masked face priming. NeuroImage, 40, 884-895.

Huber, D. E. (2008). Immediate priming and cognitive aftereffects. Journal of Experimental Psychology: General, 137, 324-347.

Huber, D. E., \& O'Reilly, R. C. (2003). Persistence and accommodation in short-term priming and other perceptual paradigms: Temporal segregation through synaptic depression. Cognitive Science, 27, 403-430.

Huber, D. E., Shiffrin, R. M., Lyle, K. B., \& Ruys, K. I. (2001). Perception and preference in short-term word priming. Psychological Review, 108, 149-182.

Jacoby, L. L., Kelley, C., Brown, J., \& Jasechko, J. (1989). Becoming famous overnight: Limits on the ability to avoid unconscious influences of the past. Journal of Personality and Social Psychology, 56, 326-338.

Jiang, Y., Costello, P., \& He, S. (2007). Processing of invisible stimuli: advantage of upright faces and recognizable words in overcoming interocular suppression. Psychological Science, 18, 349-355.

Jiang, Y., \& He, S. (2006). Cortical responses to invisible faces: dissociating subsystems for facial-information processing. Current Biology, 16, 2023-2029.

Kanwisher, N., \& Yovel, G. (2006). The fusiform face area: A cortical region specialized for the perception of faces. Philosophical Transactions of the Royal Society Bi, 361, 2109-2128.

Kornmeier, J., Hein, C. M., \& Bach, M. (2009). Multistable perception: When bottom-up and top-down coincide. Brain and Cognition, 69, 138-147.

Kouider, S., Berthet, V., \& Faivre, N. (2011). Preference is biased by crowded facial expressions. Psychological Science, 22, 184-189.

Kouider, S., \& Dehaene, S. (2007). Levels of processing during nonconscious perception: A critical review of visual masking. Philosophical Transactions of the Royal Society B, 362, 857-875.

Kouider, S., \& Dupoux, E. (2007). How "semantic" is response priming restricted to practiced items? A reply to Abrams \& Grinspan (2007). Consciousness and Cognition, 16, 954-956.
Kouider, S., Eger, E., Dolan, R., \& Henson, R. N. (2009). Activity in face-responsive brain regions is modulated by invisible, attended faces: Evidence from masked priming. Cerebral Cortex, 19, 13-23.

Lin, Z., \& He, S. (2009). Seeing the invisible: the scope and limits of unconscious processing in binocular rivalry. Progress in Neurobiology, 87, 195-211.

Lleras, A., \& Enns, J. T. (2004). Negative compatibility or object updating? A cautionary tale of mask-dependent priming. Journal of Experimental Psychology. General, 133, 475-493.

Lleras, A., \& Enns, J. T. (2005). Updating a cautionary tale of masked priming: Reply to Klapp (2005). Journal of Experimental Psychology. General, 134, 436-440.

Lleras, A., \& Enns, J. T. (2006). How much like a target can a mask be? Geometric, spatial, and temporal similarity in priming: A reply to Schlaghecken and Eimer (2006). Journal of Experimental Psychology: General, 135, 495-500.

Marcel, A. J. (1983). Conscious and unconscious perception: Experiments on visual masking and word recognition. Cognitive Psychology, 15, 197-237.

Pearson, J., \& Brascamp, J. (2008). Sensory memory for ambiguous vision. Trends in Cognitive Sciences, 12, 334-341.

Rieth, C. A., \& Huber, D. E. (2010). Priming and habituation for faces: Individual differences and inversion effects. Journal of Experimental Psychology: Human Perception and Performance, 36, 596-618.

Seitz, A. R., Kim, D., \& Watanabe, T. (2009). Rewards evoke learning of unconsciously processed visual stimuli in adult humans. Neuron, 61, 700-707.

Shin, K., Stolte, M., \& Chong, S. C. (2009). The effect of spatial attention on invisible stimuli. Attention, Perception, \& Psychophysics, 71, $1507-1513$.

Sterzer, P., Haynes, J. D., \& Rees, G. (2008). Fine-scale activity patterns in high-level visual areas encode the category of invisible objects. Journal of Vision, 8(15, Art. 10), 1-12.

Sterzer, P., Jalkanen, L., \& Rees, G. (2009). Electromagnetic responses to invisible face stimuli during binocular suppression. NeuroImage, 46, 803-808.

Sumner, P. (2007). Negative and positive masked-priming - implications for motor inhibition. Advances in Cognitive Psychology, 3, 317326.

Sumner, P., Tsai, P. C., Yu, K., \& Nachev, P. (2006). Attentional modulation of sensorimotor processes in the absence of perceptual awareness. Proceedings of the National Academy of Sciences, 103, $10520-10525$.

Tong, F., Meng, M., \& Blake, R. (2006). Neural bases of binocular rivalry. Trends in Cognitive Sciences, 10, 502-511.

Tsuchiya, N., \& Koch, C. (2005). Continuous flash suppression reduces negative afterimages. Nature Neuroscience, 8, 10961101.

Tsuchiya, N., Koch, C., Gilroy, L. A., \& Blake, R. (2006). Depth of interocular suppression associated with continuous flash suppression, flash suppression, and binocular rivalry. Journal of Vision, 6 (10, Art. 6), 1068-1078.

Vuilleumier, P., Henson, R. N., Driver, J., \& Dolan, R. J. (2002). Multiple levels of visual object constancy revealed by eventrelated fMRI of repetition priming. Nature Neuroscience, 5, 491499.

Wentura, D., \& Frings, C. (2005). Repeated masked category primes interfere with related exemplars: New evidence for negative semantic priming. Journal of Experimental Psychology: Learning, Memory, and Cognition, 31, 108-120.

Wolfe, J. M. (1984). Reversing ocular dominance and suppression in a single flash. Vision Research, 24, 471-478.

Yang, E., Hong, S. W., \& Blake, R. (2010). Adaptation aftereffects to facial expressions suppressed from visual awareness. Journal of Vision, 10(12, Art. 24), 1-13. 